\title{
EXISTENCE OF BEST PROXIMITY POINTS ON GEOMETRICAL PROPERTIES OF PROXIMAL SETS
}

\author{
Arul Ravi $\mathbf{S}^{1}$, Eldred AA ${ }^{2}$ \\ ${ }^{1,2}$ Assistant Professor, \\ Department of Mathematics, St. Xavier's College (Autonomous), \\ Palayamkottai \\ ammaarulravi@gamil.com
}

Article History:Received:11 november 2020; Accepted: 27 December 2020; Published online: 05 April 2021

ABSTRACT : The notion of proximal intersection property and UC property is used to establish the existence of the best proximity point for mappings satisfying contractive conditions.

Keywords: Best Proximity point, Proximal sets, UC property, proximal intersection property.

\section{Introduction and Preliminaries:}

Let $X$ be a nonempty set and $T$ be a self map of $X$. An element $x \in X$ is called a fixed point of $T$ if $T x=x$. Fixed point theorems deal with sufficient conditions on $X$ and $T$ ensures the existence of fixed points. Suppose the fixed point equation $T x=x$ does not posses a solution, then the natural interest to find an element $x \in X$, such that $x$ is in proximity to $T x$ in some cases.

In other words we would like to get a desirable estimate for the quality $d(x, T x)$.

It is natural that some mapping, especially non-self mappings defined on a metric space $(X, d)$, do not necessarily possess a fixed point that $d(x, T x)>0$ for all $x \in X$. In such situations, it is reasonable to search for the existence and uniqueness of the point $x \in X$ such that $d(x, T x)=0$.

In other words, one intends to determine an approximate solution $x \in X$ that is optimal in the sense that the distance between $x$ and $T x$ is minimum. Here the point $x$ is the proximity point. That is $d(x, T x)=d(A, B)$ where $d(A, B)=\inf \{d(x, y): x \in A, y \in B\}$.

In Suzuki et al [1], UC property was introduced to prove some existence results on best proximity point. In Raj and Eldred [2], the author introduced $p$-property and proved strict convexity is equivalent to $p-$ property.

We use proximal intersection property for a pair $(A, B)$ where $A$ and $B$ are non empty closed subsets of a metric space. Then this property is used to prove the existence of the best proximity point for mapping satisfying some contractive conditions introduced by Wong [3].

In this section, we use some basic definitions and concepts that are related to the context of our main results.

Definition:1.1 [4] Let $A$ and $B$ be nonempty subsets of a metric space $(X, d)$. Then, the pair $(A, B)$ is said to satisfy the property UC if the following holds: If $x_{n}$ and $x_{n}^{\prime}$ are sequence in $A$ and $y_{n}$ is a sequence in $B$ such that $\lim _{n \rightarrow \infty} d\left(x_{n}, y_{n}\right)=d(A, B)$ and $\lim _{n \rightarrow \infty} d\left(x_{n}^{\prime}, y_{n}\right)=d(A, B)$ then $\lim _{n \rightarrow \infty} d\left(x_{n}, x_{n}^{\prime}\right)=0$ holds.

Definition:1.2 Let $A$ and $B$ be nonempty subsets of a metric space $(X, d)$. Then $(A, B)$ is said to satisfy proximal intersection property if whenever $A_{n} \subset A$ and $B_{n} \subset B$ are a decreasing sequence of closed subsets such that $\delta(A, B) \rightarrow d(A, B)$, then $\cap A_{n}=\{x\}, \cap B_{n}=\{y\}$ with $d(x, y)=d(A, B)$.

Remark:1.1 $d(A, B) \rightarrow d(\bar{A}, \bar{B})$ and $\delta(A, B) \rightarrow d(\bar{A}, \bar{B})$ where $\delta(A, B)=\operatorname{Sup}\{\|x-y\|: x \in A, y \in B\}$. Definition:1.3 [2] Let $X$ be a metric space and let $T: X \rightarrow X$. Then $d_{T}$ is the function on $X \times X$ defined by $d_{T}(x, y)=\inf \left\{d\left(T_{x}^{n}, T_{y}^{n}\right): n \geq 1, x, y \in X\right\}$

Definition:1.4 [3] Let $A$ and $B$ be nonempty subsets of a metric space $X$. We shall use $X_{d}$ to denote the set $\left\{r^{\prime}\right.$ : for some $s>r^{\prime}, d(x, y)-d(A, B) \in\left[r^{\prime}, s\right]$ for some $\left.x \in A, y \in B\right\}$.

Remark:1.2 If $r^{\prime} \in X_{d}$, then there exists $x_{n} \in A, y_{n} \in B$ such that $d\left(x_{n}, y_{n}\right)-d(A, B) \rightarrow r^{\prime}$. Also if $x \in A, y \in$ $B$, then $d\left(x_{n}, y_{n}\right)-d(A, B) \in X_{d}$ and if $x_{n} \in A, y_{n} \in B$ such that $d\left(x_{n}, y_{n}\right)-d(A, B) \rightarrow r^{\prime}$, then $r^{\prime} \in X_{d}$.

Lemma:1.1 [1] Let $A$ and $B$ be nonempty subsets of a metric space $(X, d)$. Then $(A, B)$ has the property UC. Let $\left\{x_{n}\right\}$ and $\left\{y_{n}\right\}$ be sequence in $A$ and $B$ respectively such that either of the following holds:

$\lim _{m \rightarrow \infty} \operatorname{Sup}_{n \geq m} d\left(x_{m}, y_{n}\right)=d(A, B)$ or

$\lim _{n \rightarrow \infty} \operatorname{Sup}_{m \geq n} d\left(x_{m}, y_{n}\right)=d(A, B)$

Then $\left\{x_{n}\right\}$ is Cauchy.

*Corresponding author: Arul Ravi S

Assistant Professor,

Department of Mathematics, St. Xavier's College (Autonomous),

Palayamkottai

ammaarulravi@gamil.com 


\section{Results:}

Theorem:2.1 Let $A$ and $B$ be nonempty closed subsets of a complete metric space $X$ satisfying UC property. Let $A_{n}, B_{n}$ be decreasing sequence of nonempty closed subsets of $X$ such that $\delta\left(A_{n}, B_{n}\right) \rightarrow d(A, B)$ as $n \rightarrow \infty$ then $\cap A_{n}=\{x\}, \cap B_{n}=\{y\}$ with $d(A, B)$ that is $(A, B)$ satisfies proximal intersection property.

Proof: Construct a sequence $x_{n}, y_{n}$ in $X$ by selecting $x_{n} \in A_{n}, y_{n} \in B_{n}$ for each $n \in N$.

Since $A_{n+1} \subseteq A_{n}, B_{n+1} \subseteq B_{n}$ for all $n$, we have $x_{n} \in A_{n} \subseteq A_{m}, y_{n} \in B_{n} \subseteq B_{m}$ for all $n>m$.

We claim that $x_{n}$ is a Cauchy sequence.

Let $\varepsilon>0$ be given.

Since $\delta\left(A_{n}, B_{n}\right) \rightarrow d(A, B)$, there exists a positive integer $N$ such that $\delta\left(A_{n}, B_{n}\right)<d(A, B)+\varepsilon$, for all $n \geq N$.

Since $A_{n}, B_{n}$ are decreasing sequence, we have $A_{n}, A_{m} \subseteq A_{N}$ and $B_{n}, B_{m} \subseteq B_{N}$ for all $m, n \geq N$.

therefore $x_{n}, x_{m} \in A_{N}$ and $y_{n}, y_{m} \subseteq B_{N}$ for all $m, n \geq N$,

and there we have

$d\left(x_{n}, x_{m}\right) \leq \delta\left(A_{n}, B_{n}\right)<d(A, B)+\varepsilon$, for all $m, n \geq N$

since $A$ and $B$ satisfy UC property from lemma $1.1, x_{n}$ is a cauchy sequence. There exists $x \in A$ such that $x_{n} \rightarrow$ $x$.

similarly there exists $y \in B$ such that $y_{n} \rightarrow y$

we claim that $x \in \cap A_{n}, y \in \cap B_{n}$,

since $A_{n}$ and $B_{n}$ are closed for each $n$,

$x \in A_{n}, y \in B_{n}$ for all $n \in N$

since $d\left(x_{n}, y_{n}\right) \rightarrow d(A, B)$ we have

$d(x, y)=d(A, B)$

finally to establish that $x$ is the only point in $\cap A_{n}$, if $x_{1} \neq x_{2} \in \cap A_{n}$, then $d(x, y)=d(A . B)$ UC property forces that $x_{1}=x_{2}$, similarly $\cap B_{n}=\{y\}$.

Lemma:2.1

(i) Let $A$ and $B$ be nonempty closed subsets of a complete metric space $X$ such that $(A, B)$ satisfying UC property. Let $T: A \cup B \rightarrow A \cup B$ be continuous, suppose that $T(A) \subset B, T(B) \subset A$ be a continuous function such that $\inf \{d(x, T x): x \in A\}=d(A, B)=\inf \{d(x, T x): x \in A\}=d(A, B)$ There exists $\delta_{n}>0$ such that $\mathrm{d}(\mathrm{Tx}, \mathrm{Ty})-d(A, B)<\frac{1}{n} \quad$ whenever $\max \{\mathrm{d}(\mathrm{x}, \mathrm{Tx})-$ $d(A, B), d(y, T y)-d(A, B)\}<\delta_{n}$ and $x \in A^{\prime}, y \in B^{\prime}$ where $A^{\prime}$ and $B^{\prime}$ are any closed bounded sets of $A$ and $B$ respectively.

Then, there exists a best proximity point $x \in A$, such that $d(x, T x)=d(A, B)$, Further, if $d(T x, T y)=$ $d(x, y)$ for all $x \in A, y \in B$ then the best proximity point is unique.

Proof: Let $A_{n}=\left\{x \in A: d(x, T x)-d(A, B) \leq \frac{1}{n}\right\}$

$B_{n}=\left\{y \in B: d(y, T y)-d(A, B) \leq \frac{1}{n}\right\}$ since $T$ is continuous, $A_{n}, B_{n}$ are closed

from (i) $A_{n}$ and $B_{n}$ are nonempty

there exists $\mathrm{N}$ for all $n \in N$

let $x \in A_{n}, y \in B_{n}$

then $d(x, T x)-d(A, B)<\delta_{n}$ and

$d(y, T y)-d(A, B)<\delta_{n}$

from (ii) $d(T x, T y)-d(A, B) \leq \frac{1}{n}$ where $\delta_{n} \rightarrow 0$

for any $x \in A_{n}, y \in B_{n}$

$d(T x, T y)-d(A, B) \leq \frac{1}{n}$

which implies $\delta\left(T\left(A_{n}\right), T\left(B_{n}\right)\right) \rightarrow d(A, B)$

and hence $\delta\left(\overline{T\left(A_{n}\right)}, \overline{\left.T\left(B_{n}\right)\right)} \rightarrow d(A, B)\right.$

By proximal intersection property,

we have $\bigcap_{n \geq 1} \overline{T\left(A_{n}\right)}=y$ and $\bigcap_{n \geq 1} \overline{T\left(B_{n}\right)}=x$ and

$d(x, y)=d(A, B)$

Thus for each $n \geq 1$, there exists $x_{n} \in A_{n}$

such that $d\left(y, T x_{n}\right)<\frac{1}{n}$

since $d\left(x_{n}, T x_{n}\right) \rightarrow d(A, B)$ and

$d\left(y_{n}, T y_{n}\right) \rightarrow d(A, B)$

By UC property $x_{n} \rightarrow x$

Since $A_{n}$ is closed, $x \in A_{n}$ for each $n$

This implies that $d(x, T x) \rightarrow d(A, B)$

Similarly $y_{n} \rightarrow y$ such that $d(y, T y) \rightarrow d(A, B)$

To prove uniqueness, 
$d(x, T x)=d(A, B)$

Since $T$ is non expansive $d\left(T^{2} x^{\prime}, T x^{\prime}\right)=d(A, B)$ which implies that $T^{2} x^{\prime}=x^{\prime}$ as

$d(x, T x)=d\left(T x^{\prime}, T^{2} x^{\prime}\right)=d(A, B)$

from (ii) $d\left(T x, x^{\prime}\right)=d\left(T x, T^{2} x^{\prime}\right)=d(A, B)$

which implies that $x=x^{\prime}$

Theorem:2.2 Let $A$ and $B$ be nonempty closed subsets of a metric space $X$ and let Let $T: A \cup B \rightarrow A \cup B$ be continuous, such that $T(A) \subset B, T(B) \subset A$. Suppose that there exists $\phi: X_{d} \rightarrow[0, \infty)$ such that $d(x, y)-$ $d(A, B) \leq \phi((x, y)-d(A, B))$ for all $x \in A, y \in B$ and $\sup _{\delta>r} i n f_{t \in[r, s]}(t-\phi(t))>0$ for $r \in X_{d}-\{0\}$. Then $d_{T}(x, y)=d(A, B)$ for all $x \in A, y \in B$ hence $\inf \{d(x, T x): x \in A\}=d(A, B)$

Proof: Suppose to the contrary that there exists $x \in A, y \in B$ such that

Inf $\left\{d\left(T^{n} x, T^{n} y\right): n \geq 1\right\}>d(A, B)$.

by hypothesis there exists $s \in\left(r^{\prime}, \infty\right)$ such that $u=i n f_{t \in\left[r^{\prime}, s\right]}(t-\phi(t))>0$ where $r^{\prime}=r-d(A, B)$

since there exists a sequence

$d\left(T^{n} x, T^{n} y\right)-d(A, B) \rightarrow r^{\prime}$ where $r^{\prime} \in X_{d}-\{0\}$

Then from (2) we have

$d\left(T^{n} x, T^{n} y\right)-d(A, B) \rightarrow r^{\prime}+t<s$ for some $n \geq 1$.

Since $d\left(T^{n} x, T^{n} y\right)-d(A, B) \in\left[r^{\prime}, s\right]$

$u \leq d\left(T^{n} x, T^{n} y\right)-d(A, B)-\phi\left(d\left(T^{n} x, T^{n} y\right)-d(A, B)\right)$

$\phi\left(d\left(T^{n} x, T^{n} y\right)-d(A, B)\right) \leq d\left(T^{n} x, T^{n} y\right)-d(A, B)-u$

If $T^{n} x \in A, T^{n} y \in B$ and vice versa

It follows that

$d_{T}(x, y)-d(A, B) \leq d_{T}\left(T^{n} x, T^{n} y\right)-d(A, B)$

$\leq d\left(T^{n} x, T^{n} y\right)-d(A, B)$

$\leq \phi\left(d\left(T^{n} x, T^{n} y\right)-d(A, B)\right)$

$\leq d\left(T^{n} x, T^{n} y\right)-d(A, B)$ from $(5)$.

$<r^{\prime}+t-u$.

Letting $\mathrm{t} \rightarrow 0$, we have

$d_{T}(x, y)-d(A, B) \leq r^{\prime}-u$

$d_{T}(x, y)-d(A, B) \leq r^{\prime}-d(A, B)-u$

$d_{T}(x, y) \leq r-u$ a contradiction.

Theorem:2.3 Let $A$ and $B$ be nonempty closed subsets of a metric space $X$. Suppose $(A, B)$ satisfies UC property. Let $T$ be as in theorem 2.2 then $T$ satisfies all the conditions of lemma 2.1 and therefore $T$ has a unique best proximity point.

Proof: Clearly from theorem 2.2 and (i) 2.1 of lemma are satisfied.

To prove (ii) of lemma 2.1 assume $x_{n} \in A$, and $y_{n} \in B$ are bounded sequences, then $d\left(x_{n}, T x_{n}\right)$ and $d\left(y_{n}, T y_{n}\right) \rightarrow d(A, B)$ where $x_{n}$ and $y_{n}$ are sequences in $A$ and $B$ resoectively.

suppose $d\left(x_{n}, T x_{n}\right)-d(A, B) \rightarrow 0$

since $x_{n}, y_{n}$ are bounded sequence, there exists subsequence $n_{k}$ and $r>0$ such that $d\left(T x_{n_{k}}, T y_{n_{k}}\right)-$ $d(A, B) \rightarrow r>0$

clearly $r \in X_{d}$

let $r_{n_{k}}=d\left(T x_{n_{k}}, T y_{n_{k}}\right)-d(A, B)$ and

$s_{n_{k}}=d\left(x_{n_{k}}, y_{n_{k}}\right)-d(A, B)$

given $r_{n_{k}}-s_{n_{k}} \rightarrow 0$ as $k \rightarrow \infty$

$d\left(T x_{n_{k}}, T y_{n_{k}}\right)-d(A, B) \leq d\left(T x_{n_{k}}, T y_{n_{k}}\right)-d(A, B)$

therefore $r_{n_{k}} \leq \phi\left(s_{n_{k}}\right)$.

now from (13) we have

$0>\phi\left(s_{n_{k}}\right)-s_{n_{k}}$

$=\phi\left(s_{n_{k}}\right)-r_{n_{k}}+r_{n_{k}}-s_{n_{k}}$

$\geq r_{n_{k}}-s_{n_{k}}$

since $r_{n_{k}}-s_{n_{k}} \rightarrow 0$ we have

$\liminf f\left(\phi\left(s_{n_{k}}\right)-s_{n_{k}}\right)=0$

contradicting inf $f_{t \in\left[r_{0}, s\right]}(t-\phi(t))>0$ where $s_{n_{k}} \rightarrow r_{0}$.

This completes the proof.

\section{References:}

Suzuki T,Kikkawa M,Vefro C. The existence of best proximity points in metric spaces with the UC property UC.Nonlinear Anal 2009;71:2918-26. 
Raj VS,Eldred A.A characterization of strictly convex space and applications. J optim theory Appl 2014;160:703-10.

Woug CS.Fixed point theorems for non-expansive mappings. J Math Anal Appl 1972;37:142-50.

Eldred A. Ph.D thesis. Madras:Indian Institutes of Technology; 2007. 